\begin{tabular}{|c|l|}
\hline Title & Targeted gene delivery in the cricket brain, using in vivo electroporation \\
\hline Author(s) & $\begin{array}{l}\text { Matsumoto, Chihiro Sato; Shidara, Hisashi; Matsuda, Koji; Nakamura, Taro; Mito, Taro; Matsumoto, Y ukihisa; Oka, } \\
\text { Kotaro; Ogawa, Hiroto }\end{array}$ \\
\hline Citation & $\begin{array}{l}\text { Journal of Insect Physiology, 59(12), 1235-1241 } \\
\text { https:/doi.org/L0.1016/.jinsphys.2013.10.001 }\end{array}$ \\
\hline Issue Date & 2013 12 \\
\hline Doc URL & http://hdl.handle.net/2115/53649 \\
\hline Type & article (author version) \\
\hline File Information & main_text_final.pdf \\
\hline
\end{tabular}

Instructions for use 


\section{Targeted gene delivery in the cricket brain, using in vivo electroporation}

Chihiro Sato Matsumoto ${ }^{\mathrm{a}}$, Hisashi Shidara ${ }^{\mathrm{b}}, \mathrm{Koji} \mathrm{Matsuda}^{\mathrm{c}}$, Taro Nakamura ${ }^{\mathrm{c}}$, Taro Mito ${ }^{\mathrm{c}}$, Yukihisa Matsumoto $^{\mathrm{a}, \dagger}$, Kotaro Oka ${ }^{\mathrm{b}}$ and Hiroto Ogawa ${ }^{\mathrm{a}, \mathrm{d}, *}$

$>$ Family name in "Chihiro Sato Matsumoto" is "Matsumoto" (Matsumoto CS)

${ }^{a}$ Department of Biological Science, Faculty of Science, Hokkaido University, Kita 10 Nishi 8, Kita-ku, Sapporo 060-0819, Japan

${ }^{\mathrm{b}}$ Department of Biosciences and Informatics, Faculty of Science and Technology, Keio University, 3-14-1 Hiyoshi, Kohoku-ku, Yokohama 223-8522, Japan

${ }^{\mathrm{c}}$ Department of Life Systems, Institute of Technology and Science, University of Tokushima, 2-1 Minami-Jyousanjima-cho, Tokushima 770-8506, Japan

d JST, PREST, 4-1-8 Honcho Kawaguchi, Saitama, 332-0012, Japan

$\uparrow$ Present affiliation: Department of Biology, College of Liberal Arts and Sciences, Tokyo Medical and Dental University, Kohnodai, Ichikawa, Chiba 272-0827, Japan

\section{•*Corresponding author:}

\section{Hiroto Ogawa}

e-mail address: hogawa@sci.hokudai.ac.jp

Postal address: Department of Biological Science, Faculty of Science, Hokkaido University, Kita 10 Nishi 8, Kita-ku, Sapporo 060-0810, Japan

Phone /Fax : +81-11-706-3525 


\begin{abstract}
The cricket (Gryllus bimaculatus) is a hemimetabolous insect that is emerging as a model organism for the study of neural and molecular mechanisms of behavioral traits. However, research strategies have been limited by a lack of genetic manipulation techniques that target the nervous system of the cricket. The development of a new method for efficient gene delivery into cricket brains, using in vivo electroporation, is described here. Plasmid DNA, which contained an enhanced green fluorescent protein (eGFP) gene, under the control of a G. bimaculatus actin $\left(G b^{\prime}\right.$-act) promoter, was injected into adult cricket brains. Injection was followed by electroporation at a sufficient voltage. Expression of eGFP was observed within the brain tissue. Localized gene expression, targeted to specific regions of the brain, was also achieved using a combination of local DNA injection and fine arrangement of the electroporation electrodes. Further studies using this technique will lead to a better understanding of the neural and molecular mechanisms that underlie cricket behaviors.
\end{abstract}

\title{
Keywords
}

Insect; Cricket; Brain; Electroporation; Neuron; Green Fluorescent Protein

\section{Abbreviations}

eGFP, enhanced green fluorescent protein ; PP, poring pulse; TP, transfer pulse; SR, success rate 


\section{Introduction}

The cricket, Gryllus bimaculatus, is one of the classic model organisms used for studying neural mechanisms that underlie the behavioral traits of insects. Most studies, for example, those on escape behavior, phonotaxis, aggression and associative learning, have relied on electrophysiological, pharmacological and neuroimaging methods (Hedwig, 2006; Matsumoto and Mizunami, 2002; Ogawa et al., 2006; 2008; Stevenson and Rillich, 2012). In spite of the many studies into a broad repertoire of behavior, the molecular basis that underlies these traits is still unclear. This may be attributed to the existence of very few established techniques for the genetic manipulation of neurons in the cricket. The availability of a simple and effective method to introduce exogenous genes into the brains of living adult crickets will accelerate biological studies in a variety of fields.

There have been several successful attempts to introduce exogenous genes and other constructs into crickets (Zhang et al., 2002; Shinmyo et al., 2004; Nakamura et al., 2010; Mito et al., 2010; Watanabe et al., 2012). In these studies, which were performed to clarify development and regeneration mechanisms, eggs or nymphs were the main targets of gene transfer. In order to study the effects of genetic manipulation in adult crickets, the eggs and nymphs need to be reared to adulthood. The establishment of a simple and efficient method of gene delivery into post-mitotic cells of adult crickets would bypass the rearing of genetically manipulated eggs and nymphs, to allow the reduction of experimental time and resources.

Electroporation has been established as a powerful means of gene transfer and is applicable to post-mitotic cells. The electroporation method, which was initially invented for gene transfer in in vitro cultured cells (Neumann et al., 1982), has been successfully adapted to in vivo gene transfer in various species. It has been used in Drosophila (Kamdar et al., 1995), honeybee (Kunieda and Kubo, 2004), zebrafish (Buono et al., 1992), Xenopus (Eide et al., 2000), chick 
(Yasuda et al., 2000; Yasugi and Nakamura, 2000; Swartz et al., 2001) and mouse (Akamatsu et al., 1999; Miyasaka et al., 1999). Gene delivery by electroporation has been tested on a wide range of targets, from single cells (Haas et al., 2001) to tissues. However, the honeybee is currently the only insect in which gene transfer by electroporation has been carried out on the adult brain of a living insect (Kunieda and Kubo, 2004). Here, we describe a method for simple in vivo electroporation, which led to the expression of enhanced green fluorescent protein (eGFP) within the brains of living adult crickets. This study is the first report of successful transfer of a foreign gene into neural tissue of adult hemimetabolous insects. We also developed a novel method to target the delivery to specific regions within the brain, without the use of a cell-specific promoter. This was achieved by combining local microinjection of DNA with a fine arrangement of the electroporation electrodes. These techniques have the potential to extend the functional analysis of cricket behaviors.

\section{Materials and methods}

\subsection{Animals}

Adult male crickets (Gryllus bimaculatus) from colonies maintained at Hokkaido University were used within 2 weeks from their imaginal molt. The animals were fed on a compounded diet for insects (Oriental Yeast, Tokyo, Japan) and were kept in a 12/12 h light/dark cycle at a constant temperature of $28^{\circ} \mathrm{C}$. Crickets were transferred from the colony to the laboratory environment during the week before the experiment. To avoid cannibalization, they were isolated in individual plastic cups with lids. To reduce body fluid, crickets were deprived of water for at least 3 days before the experiment. This allows easier fluid control and improves the success rate of plasmid injection and electroporation. During isolation, crickets were fed on insect pellets, ad libitum, as in the rearing environment. 


\subsection{Plasmids}

To construct the plasmid DNA used for electroporation, the promoter of the G. bimaculatus cytoplasmic actin $\left(G b^{\prime}-a c t\right)$ gene, the eGFP gene (derived from pEGFP-N1; Clontech Laboratories Inc., Mountain View, USA) and 3' untranslated region (UTR), which contained the SV40 polyadenylation signal, were PCR amplified from the plasmid pGact-eGFP (Zhang et al., 2002). The PCR was performed with a sense primer that contained an XbaI site (5'-GCTCTAGAGCGGCCGCCACCGC-3') and an antisense primer that contained an EcoRI site (5'-GGAATTCAGGGGGGGCCCGGTACCCAA-3'). The $G b^{\prime}$-act promoter is an 897 base pair (bp) genomic DNA fragment that is found upstream of the putative start codon of the $G b^{\prime}$-act gene (accession number: AB087882.1). Its strong activity in G. bimaculatus embryonic cells has been reported previously (Zhang et al., 2002; Shinmyo et al., 2004; Nakamura et al., 2010). A $G b^{\prime}$-act promoter-eGFP fragment was subcloned into a vector derived from a C. elegans plasmid, pttx-3::GFP (pPD95.75 vector; Tsalik and Hober, 2003), using the XbaI and EcoRI sites. The total length of the plasmid was $5606 \mathrm{bp}$ and is shown in Figure 1A. The plasmid was extracted and purified in distilled water using the QIAGEN Plasmid Plus Maxi Kit (QIAGEN, Hamburg, Germany). For the expression of Yellow Cameleon 3.60 (YC3.60, Nagai et al., 2004) that is a genetically encoded $\mathrm{Ca}^{2+}$ indicator (GECI) and Channel Rhodopsin 2 (ChR2, Nagel et al., 2003) that is a light-activated cation channel, plasmids $G b^{\prime}$-act-YC3.60-SV40 and $G b^{\prime}$-act-ChR2-DsRed2-SV40 were constructed by replacing the eGFP sequence with either YC3.60 or ChR2-DsRed2. The ChR2 gene and DsRed2 gene were used for observation of ChR2 expression (pDsRed2-C1 vector, Clontech 6974-1). The $G b^{\prime}$-act-YC3.60-SV40 and $G b^{\prime}$-act-ChR2-DsRed2-SV40 plasmids were 6388 bp and 6503 bp, respectively. 


\subsection{Preparation.}

For plasmid injection and electroporation, crickets were anesthetized by cooling on crushed ice. They were individually fixed with a harness set that consisted of a neck holder and an abdomen holder. The neck holder was made from the lid of small acrylic petri dish with a keyhole-shaped hole. Two pieces of plastic tape of $\sim 18 \mathrm{~mm}$ by $20 \mathrm{~mm}$ and a third piece of $\sim 18$ $\mathrm{mm}$ by $25 \mathrm{~mm}$, with a square hole in the center ( $4 \mathrm{~mm}$ by $4 \mathrm{~mm}$ ), were used to harness a cricket to the neck holder. A $1.5 \mathrm{ml}$ microtube was used as the abdomen holder, together with a piece of dental utility wax (GC Corp., Tokyo, Japan). The head of the anesthetized cricket was pushed through the hole in the neck holder, with the neck set at the narrow part of the keyhole. The rest of the hole was covered with a piece of plastic tape behind the neck of the cricket. The other piece of tape was used to immobilize the antennae. The piece of tape with the square hole was used to cover and stabilize the head, with the necessary part of the head exposed for access to the brain (Fig. 1B). The abdomen was placed in the abdomen holder, which was attached to the neck holder by the piece of dental utility wax. This prevented the body from twisting at the neck, which could cause fatal damage. For dissection, injection and electroporation, the neck holder was set on a tubular platform.

\subsection{Plasmid injection}

The plasmid DNA was manually pressure-injected using a glass needle connected to a disposable syringe. Glass needles were prepared from borosilicate glass capillary tubes (1B100-4, World Precision Instruments, Sarasota, USA) using a micropipette puller (PN-3, Narishige, Tokyo, Japan). The tips were broken to obtain a point diameter of approximately 10-15 $\mu \mathrm{m}$. To expose the frontal surface of the brain, part of the head cuticle was carefully 
removed and the tissues covering the brain were pushed aside. The neural sheath that covers the brain was not removed. To deliver the DNA into the whole brain, a glass needle was filled with $200 \mathrm{nl}$ of plasmid solution (250 ng/ $\mu$ l DNA, 0.01\% Fast Green FCF in distilled water), which had been measured out with a micropipette (NPX-2, NICHIRYO, Koshigaya, Japan). Micromanipulators (MMN-1 and MMO203, Narishige, Tokyo, Japan) were used to insert the glass needle into the brain at the precise injection site. Injection of the entire volume within the needle was visually checked under a stereomicroscope (SMZ645, Nikon, Tokyo, Japan). For local microinjection, a glass capillary $(\varnothing=1.0 \mathrm{~mm})$ was filled with $100 \mathrm{nl}$ of solution, which was measured out with a micropipette. Half of the volume $(50 \mathrm{nl})$ was sucked from the capillary into a glass needle using a disposable syringe. Slightly less than half the volume $(\sim 20 \mathrm{nl})$ was injected into the targeted region to avoid injecting air into the brain. Three regions of the protocerebrum were targeted; the medial site close to the central-body complex, the antero-lateral site close to the mushroom-body calyx and the postero-lateral site close to the antennal lobe.

A pair of platinum wires $(\varnothing=0.1 \mathrm{~mm})$ was placed on either side of the targeted brain region in advance of the plasmid injection. Immediately after the plasmid injection, one poring pulse (PP; duration: $0.1 \mathrm{~ms}$, voltage: $25-100 \mathrm{~V}$ ), followed by five transfer pulses (TPs; pulse duration: $50 \mathrm{~ms}$, frequency: $1 \mathrm{~Hz}$, voltage: 10-50 V), were delivered at defined voltages using an electroporator (NEPA21, NEPA GENE, Ichikawa, Japan; Fig. 1B). After electroporation, the head cuticle was replaced into the original position. In some cases a small volume of cricket saline was placed onto the brain to prevent drying out. Crickets were left in the harness until the head cuticles stabilized. They were then released and left in individual plastic cups overnight. The following day, the whole brain was dissected and observed using stereoscopic fluorescence microscopy (MZ16F, Leica Microsystems, Wetzlar, Germany), with the following filter 
combination: excitation 480/40; emission LP510 (Leica, GFP2).

\section{Results}

\subsection{Gene transfer by whole brain electroporation}

Our first aim was to establish a simple gene introduction protocol for adult cricket brains. Preliminary experiments on seventh and eighth instar nymph crickets were performed to confirm the potential of the electroporation method (data not shown).

We then investigated whether an exogenous gene could successfully be delivered into the adult cricket brain using in vivo electroporation. To target the whole frontal brain, the tips of platinum electrodes (Pt-electrodes) were put into the epicranium through small holes that had been opened in the soft cuticles at the base of each antenna (width between Pt-electrodes was $\sim 2$ $\mathrm{mm})$. This arrangement of electrodes provided a large enough radius, of sufficient electric current, to cover a wide range of the brain. Each of the electrodes was fixed to the neck harness with dental utility wax. The $G b^{\prime}$-actin:eGFP plasmid $(250 \mathrm{ng} / \mu \mathrm{l})$ was injected into the center of the brain, between the two electrodes. The use of $200 \mathrm{nl}$ was sufficient to spread the solution throughout the brain, which is enveloped in a neural sheath. To optimize the parameters for effective gene delivery, electroporation was performed with a PP at $100 \mathrm{~V}$ and five TPs at various voltages $(10,30$ or $50 \mathrm{~V})$. The expression of eGFP within the brain was observed for all three TP voltages (Fig. $1 \mathrm{C}$ and $2 \mathrm{~A}$ and $\mathrm{B}$ ). The $G b^{\prime}$-act non-specific promoter could drive the expression of eGFP in both the neuron and glia cells. In our results, eGFP fluorescence was observed in a putative nerve cell that had a neurite-like process (Fig. 1D), and no fluorescence was observed in the neural sheath.

Survival rate (percentage of live animals/total number of animals in experimental group), 1 
day after electroporation, and gene transfer rate (percentage of animals with eGFP expression/number of live animals) are shown for each TP voltage in Figure 2A. The survival rate was $100 \%$ at 10 or $30 \mathrm{~V}$ TPs and $93.94 \%$ at $50 \mathrm{~V}$. The gene transfer rate was $66.67 \%$ at $10 \mathrm{~V}$, $76.92 \%$ at $30 \mathrm{~V}$ and $93.55 \%$ at $50 \mathrm{~V}$. Most of the crickets were alive the day after electroporation and 95\% displayed normal behaviors, which included voluntary walking, antennal searching, feeding, drinking and singing (Table 1). The singing behavior is controlled by descending commands from the brain (Otto, 1971; Bentley, 1977).

The efficiency of gene introduction clearly depended on the TP voltage. A higher voltage led to a higher success rate in gene transfer. The higher TP voltage also led to the observation of a larger number of eGFP positive cells in individual brains (Fig. 2B). After obtaining this information, all subsequent experiments were performed with one $100 \mathrm{~V}$ PP followed by five 50 V TPs, with antenna-to-antenna electrode placement, unless otherwise noted. Some crickets were robust enough to live for more than 5 days after electroporation at this voltage. However, lower voltage electroporation would be preferable for behavioral experiments because of the reduced damage to the neural tissue.

Although these experiments did not address the critical parameters for distribution of eGFP positive cells, eGFP expression was mainly observed in the soma and nuclei of neurons located at both the anterior and posterior surfaces of the brain. There was no apparent bias of eGFP fluorescence against the arrangement and polarity of the electrodes or the plasmid injection site.

\subsection{Localized gene transfer}

The region of gene transfer was localized using two methods: reducing the injection volume of plasmid solution or narrowing the range of electroporation. 


\subsubsection{Local injection}

To confine gene delivery to the injection site, the plasmid volume was reduced to $20 \mathrm{nl}$. When this volume was used, the blue color of Fast Green stained only the local area around the injection site, with a diameter of approximately $400 \mu \mathrm{m}$. The average number of eGFP positive cells was also decreased (Fig. 2C). In gene delivery that targeted the mushroom body (MB), eGFP positive cells were observed near the injection site, within the ipsilateral hemisphere (Fig. 3A1 and A2). When gene delivery targeted the antennal lobe (AL), eGFP positive cells were widely distributed, despite the reduced injection volume (Fig. 3B1 and B2). To localize the electric field specifically to the AL, one of the electrodes was set at the medial-ventral position to the brain (approximate electrode distance $=1.3 \mathrm{~mm}$, Fig. 3C1). As shown in Figures $3 \mathrm{C} 1$ and $\mathrm{C} 2$, in two out of three samples, eGFP positive cells were restricted to within the AL. No fluorescence was observed in protocerebrum area around the MB. A combination of local plasmid injection and arrangement of the electrode placement is manageable and useful for localized gene expression at a targeted site of the brain.

\subsubsection{Adjustment of Pt-electrode placement}

In order to evaluate whether narrowing the range of the electric field is able to localize the expression of eGFP, the position of the electrodes was changed. In this experiment the electrode distance was reduced to approximately half that of the previous protocol $(<1 \mathrm{~mm})$ (Fig 4A and B). After injection of $200 \mathrm{nl}$ of plasmid solution, the electroporation pulses were applied with the electrode placements shown in Figure 4 (dorsal-ventral layout: A; medial-lateral layout: B, C and D). With the expectation that more eGFP positive cells would be localized within the restricted range, the previous voltages were maintained (100 V PP and $50 \mathrm{~V}$ TPs, Fig. 4A and B). Numerous eGFP positive cells were observed using these electrode layouts (over 100 cells, see 
also Fig. 2D), but, in contrast to our expectations, cells were broadly distributed over the brain. When one electrode was placed directly onto the center of the brain surface, in the medial-lateral layout, the medial region of the brain was obviously discolored near the electrode, as shown in Figures $4 \mathrm{~A}-\mathrm{C}$. It was assumed that the neural tissue was damaged by a significant level of electric current or heat applied by direct contact with the electrode. The eGFP positive cells were not found in the damaged region. The discolored region, which indicated tissue damage, was never observed in the brain when electrodes were placed around the brain (Fig. 3). These results indicated that a high electroporation current, applied through an electrode in direct contact with the brain surface, would result in neuronal injury. However, a high but sub-lethal voltage should provide efficient global gene delivery because a large number of cells at the boundary of the damaged area expressed eGFP. In general, the magnitude of the electric field is dependent on voltage of applied pulses and distance between the two electrodes. Therefore, if appropriate voltage pulses are applied, the eGFP positive cells should be restricted within a local area that corresponds to the damaged region observed in this protocol. Lower PP and TP voltages were tested with this electrode layout because similar damage was also induced by lower PP voltages followed by $50 \mathrm{~V}$ TPs (data not shown). As PP and TP voltages were lowered $(\mathrm{PP} / \mathrm{TP}=50 \mathrm{~V} / 25 \mathrm{~V}$ in Fig. 4C and 25 V/12.5 V in Fig. 3D1, D2 and Fig. 4D) the damaged area was narrowed. Significant tissue damage was not apparent when the 25 V PP/12.5 V TPs were applied and eGFP expression was restricted to the targeted hemisphere. However, gene transfer efficiency was decreased when compared with the local injection experiments.

3.3 Gene delivery of the genetically encoded calcium ion $\left(\mathrm{Ca}^{2+}\right)$ indicator and light-activated ion channel

Finally, we attempted to introduce YC3.60 and ChR2 into the cricket brain using 
electroporation. When $100 \mathrm{~V}$ PP/50 V TPs were applied to the whole brain, cells that expressed YC3.60 and ChR2-DsRed2 were observed within the brain (Fig. 5A). The gene transfer rate for YC3.60 was $80 \%(\mathrm{~N}=20)$ and that for ChR2, in preliminary experiments $(\mathrm{N}=3)$ was $100 \%$. The expression pattern of YC3.60 was similar to that of eGFP, with a wide distribution of YC3.60 positive cells on both the anterior and posterior surfaces of the brain. There were slightly fewer YC3.60 positive cells than eGFP positive cells, but numbers increased with days (Fig. 5C). Seven days after electroporation, approximately 100 cells expressed YC3.60. We hypothesized that it takes longer for the cricket neurons to express YC3.60, compared with eGFP. In contrast, ChR2-DsRed2 was expressed in only a few cells within the cricket brain (Fig. 5B). For expression of $\mathrm{ChR} 2$ in a larger number of cells the procedure may need to be modified.

\section{Discussion and conclusion}

We have developed a simple and highly effective method of in vivo gene delivery into the brains of adult crickets. In this study, which is the earliest report of this kind in hemimetabolous insects, the high survival rate and gene transfer rate should be emphasized (Fig. 2A).

Multiplication of survival rate and gene transfer rate gives the overall success rate (SR), which is the percentage of animals that express eGFP versus the total number of experimental animals. When a single PP at $100 \mathrm{~V}$ and five TPs at $50 \mathrm{~V}$ were applied to cricket brains, SR was $87.88 \%$, with an average of $\sim 70$ cells/animal observed to express eGFP. When TP voltages were lowered to 10 or $30 \mathrm{~V}$, SR remained over $65 \%(66.67 \%$ in $10 \mathrm{~V}, 76.92 \%$ in $30 \mathrm{~V})$. Gene transfer into the insect brain, using electroporation, has also been attempted in the honeybee (Kunieda and Kubo, 2004). There are two differences between the results from the cricket and the honeybee. Firstly, the overall success rate of the cricket method was considerably higher when compared with the top success rates calculated for the honeybee. The honeybee SR, with five pulses of $50 \mathrm{~V}$, was 
$31.82 \%$ (survival rate $=54.55 \%$, gene transfer rate $=58.33 \%$, and was $27.27 \%$ (survival rate $=$ $29.55 \%$, gene transfer rate $=92.31 \%$ ) with $60 \mathrm{~V}$ (Kunieda and Kubo, 2004). The change in viability could be due to the physical robustness of crickets or the use of a protocol that employed a 100 V PP plus TPs. This PP and TP procedure may enhance the gene transfer efficiency. The second difference was that the restriction of gene expression depended solely on anode placement in the honeybees (Kunieda and Kubo, 2004). However, in the cricket experiments the distribution of eGFP positive cells was not strongly biased to the anode side, but rather depended on the intensity of the electrical field (Fig. 4). The discrepancies may have resulted from improvements in the electroporation procedure. The plasmid solution was injected directly into the cricket brain, whilst in honeybees the DNA was injected to spread over the brain. The latter protocol is expected to lead to attraction of the DNA to the electrodes. However, when the DNA is injected into the brain it can easily be taken up by neighboring cells, as in the cricket experiments. Another improvement in the method used for crickets, which was not applied to the honeybee, was the use of a poring pulse. The cell membrane may need multiple pulses to create transfer pores in the honeybee, whilst a single poring pulse of higher voltage is either sufficient to form the pore in crickets or strongly supports the subsequent loading of DNA into the cells. In the case of the honeybee protocol, high voltage, multiple pulses would bias the gene expression to the vicinity of the anode position.

Although a method to produce transgenic crickets has been previously reported (Zhang et al., 2002; Nakamura et al., 2010), gene expression has not yet been targeted to the nervous system of the adult cricket. If transgenic cricket lines were established, they would provide stable expression of different foreign genes on an identical genetic background. However, development of transgenic animals, which express the genes within the desired cells and tissues, will require a huge effort over a long time period, plus the establishment of large culture plants. 
Foreign genes are found in all cells throughout the body in transgenic animals. This can cause a decrease in survival, lead to defects during development and may have an impact on the growth of the cricket. In contrast, the electroporation method is capable of rapid and efficient gene delivery into targeted brain areas. Therefore, even if the exogenous gene has some negative effects, they would be of smaller magnitude than the effects observed from inherent expression in transgenic animals. However, tissue damage can be caused by direct contact between the brain and electrode. When electrodes were placed away from the brain, the tissue discoloration, which was indicative of damage, was not observed. Electroporation with PP and TPs of low but sufficient voltage to achieve efficient gene transfer had no significant effect on cricket behaviors, which included voluntary walking, antennal searching, feeding, drinking and singing (Table 1). This observation indicated that the electroporation method described here did not have a significant, harmful effect on behavior.

Another advantage of this method is that it enables us to deliver the foreign gene into a selected region of the cricket brain without the need for a cell-specific promoter. We achieved this by combining local injection of a smaller volume of plasmid and fine arrangement of electrodes, with the appropriate voltage parameters. In summary, the following procedures were devised to localize eGFP expression from plasmids introduced into the cricket brain via electroporation: 1) injection of a small volume of plasmid DNA into the target area, which is preferably aligned within the electrodes; 2) combination of PP and TPs with sufficient but harmless voltages, whilst avoiding direct contact between electrodes and the brain.

The specificity and reproducibility of targeted gene expression was not sufficient to completely manipulate the cell function of specific neurons. Nevertheless, this method is able to efficiently target gene expression to a specific neuropile or cell cluster. If a cell-specific promoter or enhancer sequence were available, gene delivery by this method could be made 
more specific to the neurons. In addition, the combination of this technique with other cell-specific labeling methods, such as immunoreactive staining or neuronal tracing, will have other genetic applications, for example, optically-probing and manipulating neural activity.

The use of a chemical, fluorescent calcium indicator for optical imaging has been adapted for the cricket nervous system (Sobel and Tank, 1994; Ogawa et al., 2006, 2008; Baden and Hedwig, 2007, 2009). The introduction of genetically encoded voltage or calcium sensors would allow the monitoring of neural activities in specific cell types over a long period of time. In this study, we successfully expressed YC3.60 within the cricket brain. Introduction of GECI, by electroporation, will enable prolonged $\mathrm{Ca}^{2+}$ imaging in deep brain regions of the cricket. Optogenetics is another powerful tool for analyzing the neural circuits of behavior (for recent review, see Bernstein and Boyden, 2011). If light activated ion channels, such as ChR2 or archaerhodopsin, were successfully expressed in cricket neurons, it would enable the timing of neural excitation or silencing to be controlled by light. In our preliminary experiments, ChR2 was expressed in the cricket brain. However, the number of ChR2-positive cells and the reproducibility were not sufficient for practical use as optogenetic tools. Further technical progress, such as the refinement of cell-specific promoters, will provide efficient expression of a gene that encodes optogenetic molecules within targeted cells. Our electroporation method serves as the first step to provide powerful tools for the investigation of the behavioral role of specific neurons.

In conclusion, we successfully developed a convenient method for in vivo gene transfer into the brains of adult crickets. Adult crickets have been used extensively in experiments on learning and memory, the higher brain function and adult-specific behaviors (courtship, mating and aggression). It will be possible to monitor the calcium signals of a large number of Kenyon cells, which express the calcium sensor, over a long time period, before and after learning. 
Selective activation of the identified key neurons, such as the cercal giant interneurons for wind-evoked behavior or descending neurons for singing behavior, will provide direct evidence for their behavioral functions. Our gene transfer method will contribute to insect neuroscience by facilitating future and ongoing studies aimed at uncovering the neural and molecular mechanisms that underlie cricket behaviors.

\section{Acknowledgements}

We are grateful to Dr. N. Tanaka for helpful comments. This work was supported by JST, PRESTO to H.O.; JSPS and MEXT KAKENHI [24120502 to H.O.; 23687033, 22124003, and 22370080 to T.M.; \#23111521 to T.N.]; MEXT Program for the Strategic Research Foundation at Private Universities [S0801008 to K.O.] 


\section{References}

Akamatsu, W., Okano, H.J., Osumi, N., Inoue, T., Nakamura, S., Sakakibara, S., Miura, M., Matsuo, N., Darnell, R.B., Okano, H. 1999. Mammalian ELAV-like neuronal RNA-binding proteins $\mathrm{HuB}$ and $\mathrm{HuC}$ promote neuronal development in both the central and the peripheral nervous systems. Proceedings of the National Academy of Sciences of the United States of America, 96, 9885-9890.

Baden, T., Hedwig, B. 2007. Neurite specific $\mathrm{Ca}^{2+}$ dynamics underlying sound processing in an auditory interneuron. Developmental Neurobiology, 67, 68-80.

Baden, T., Hedwig, B. 2009. Dynamics of free intracellular $\mathrm{Ca}^{2+}$ during synaptic and spike activity of cricket tibial motoneurons. European Journal of Neuroscience, 29, 1357-1368.

Bentley, D.R. 1977. Control of cricket song patterns by descending interneurons. Journal of Comparative Physiology A, 116, 19-38.

Bernstein, J.G., Boyden, E.S. 2011. Optogenetic tools for analyzing the neural circuits of behavior. Trends in Cognitive Sciences, $15,592-600$.

Buono, R.J., Linser, P.J. 1992. Transient expression of RSVCAT in transgenic zebrafish made by electroporation. Molecular marine biology and biotechnology, 1, 271-275.

Eide, F.F., Eisenberg, S.R., Sanders, T.A. 2000. Electroporation-mediated gene transfer in free-swimming embryonic Xenopus laevis. FEBS Letters, 486, 29-32.

Haas, K., Sin, W.C., Javaherian, A., Li, Z., Cline, H.T. 2001. Single-cell electroporation for gene transfer in vivo. Neuron, 29, 583-91.

Hedwig, B. 2006. Pulses, patterns and paths: neurobiology of acoustic behaviour in crickets. Journal of Comparative Physiology A, 192, 677-89.

Kamdar, K.P., Wagner, T.N., Finnerty, V. 1995. Electroporation of Drosophila embryos. Methods in Molecular Biology, 48, 239-243. 
Kunieda, T., Kubo, T. 2004. In vivo gene transfer into the adult honeybee brain by using electroporation. Biochemical and Biophysical Research Communications, 318, 25-31.

Matsumoto, Y., Mizunami, M. 2002. Temporal determinants of long-term retention of olfactory memory in the cricket Gryllus bimaculatus. The Journal of Experimental Biology, 205, 1429-37.

Mito, T., Nakamura, T., Bando, T., Ohuchi, H., Noji, S. 2010. The advent of RNA interference in entomology. Entomological Science, 14: 1-8.

Miyasaka, N., Arimatsu, Y., Takiguchihayashi, K. 1999. Foreign gene expression in an organotypic culture of cortical anlage after in vivo electroporation. Neuroreport, 10, $2319-2323$

Nagai, T., Yamada, S., Tominaga, T., Ichikawa, M., Miyawaki, A. 2004. Expanded dynamic range of fluorescent indicators for $\mathrm{Ca}^{2+}$ by circularly permuted yellow fluorescent proteins., Proceedings of the National Academy of Sciences of the United States of America. 101, 10554-9.

Nagel, G., Szellas, T., Huhn, W., Kateriya, S., Adeishvili, N., Berthold, P., Ollig, D., Hegemann, P., Bamberg, E. 2003. Channelrhodopsin-2, a directly light-gated cation-selective membrane channel., Proceedings of the National Academy of Sciences of the United States of America. $100,13940-13945$.

Nakamura, T., Yoshizaki, M., Ogawa, S., Okamoto, H., Shinmyo, Y., Bando, T., Ohuchi, H., Noji, S., Mito, T. 2010. Imaging of transgenic cricket embryos reveals cell movements consistent with a syncytial patterning mechanism. Current. Biology, 20, 1641-1647.

Neumann, E., Schaefer-Ridder, M., Wang, Y., Hofschneider, P.H. 1982. Gene transfer into mouse lyoma cells by electroporation in high electric fields. The EMBO Journal, 1, 841-845. 
Ogawa, H., Cummins, G.I., Jacobs, G.A., Miller, J.P. 2006. Visualization of ensemble activity patterns of mechanosensory afferents in the cricket cercal sensory system with calcium imaging. Journal of Neurobiology, 66, 293-307.

Ogawa, H., Cummins, G.I., Jacobs, G.A., Oka, K. 2008. Dendritic design implements algorism for extraction of sensory information. The Journal of Neuroscience, 28, 4592-4603.

Otto, D. 1971. Untersuchungen zur zentralnerv.ösen Kontrolle der Lauterzeugung von Grillen. Zeitschrift für vergleichende Physiologie, 74, 227-271.

Shinmyo, Y., Mito, T., Matsushita, T., Sarashina, I., Miyawaki, K., Ohuchi, H., Noji, S. 2004. piggyBac-mediated somatic transformation of the two-spotted cricket, Gryllus bimaculatus. Development, Growth and Differentiation, 46, 343-349.

Sobel, E.C., Tank, D.W. 1994. In vivo $\mathrm{Ca}^{2+}$ dynamics in a cricket auditory neuron: an example of chemical computarion. Science, 263, 823-826.

Stevenson, P.A., Rillich, J. 2012. The decision to fight or flee - insights into underlying mechanism in crickets. Frontiers in Neuroscience, 6, 118.

Swartz, M., Eberhart, J., Mastick, G.S., Krull, C.E. 2001. Sparking new frontiers: using in vivo electroporation for genetic manipulations. Developmental Biology, 233, 13-21.

Tsalik, E.L., Hobert, O. 2003. Functional mapping of neurons that control locomotory behavior in Caenorhabditis elegans. Journal of Neurobiology, 56: 178-197

Watanabe, T., Ochiai, H., Sakuma, T., Horch, H.W., Hamaguchi, N., Nakamura, T., Bando, T., Ohuchi, H., Yamamoto, T., Noji, S., Mito, T. 2012. Non-transgenic genome modifications in a hemimetabolous insect using zinc-finger and TAL effector nucleases. Nature Communications, 3, 1017.

Yasuda, K., Momose, T., Takahashi, Y. 2000. Applications of microelectroporation for studies of chick embryogenesis. Development, Growth and Differentiation, 42, 203-206. 
Yasugi S, Nakamura H. 2000. Gene transfer into chicken embryos as an effective system of analysis in developmental biology. Development, Growth and Differentiation, 42, 195-197.

Zhang, H., Shinmyo, Y., Hirose, A., Mito, T., Inoue, Y., Ohuchi, H., Loukeris, T.G., Eggleston, P., Noji, S. 2002. Extrachromosomal transposition of the transposable element Minos in embryos of the cricket Gryllus bimaculatus. Development, Growth and Differentiation, 44, 409-17. 


\section{Figure legends}

Fig. 1. Gene transfer using in vivo electroporation. (A) Construct of the plasmid DNA used for electroporation. Blue and green boxes indicate the $G b^{\prime}$-act and eGFP regions, respectively. Amp(r) is ampicillin sequence. SV40 is the $3^{\prime}$ UTR sequence that contains the polyadenylation signals from SV40. (B) Schematic illustration of DNA injection into the entire brain. The arrangement of Pt-electrodes for subsequent whole-brain electroporation is shown. For DNA injection, a micropipette filled with plasmid DNA solution, indicated in blue, was inserted into the brain, indicated by a dashed line. DNA solution was injected manually by pressure from a syringe. The electrodes were inserted into the base of the antennae. The lower panel shows the time schedule of pulse delivery for electroporation. Immediately after the DNA injection and micropipette removal, a single poring pulse of $100 \mathrm{~V}$, with $0.1 \mathrm{~ms}$ duration, and five transfer pulses of defined voltages, with $50 \mathrm{~ms}$ duration, were applied with $950 \mathrm{~ms}$ intervals. (C, D) Fluorescent image of a cricket brain 1 day after the electroporation. Plasmid solution ( $200 \mathrm{nl})$ was injected into the brain and $100 \mathrm{~V}$ poring pulse (PP) and $50 \mathrm{~V}$ transfer pulses (TPs) were applied, with the electrodes placed as shown in B. Large numbers of eGFP positive cells were observed in the protocerebrum (C). Some of the eGFP positive cells have neurite-like process extending from the cell body (arrowhead in D).

Fig. 2. Effects of TP voltage, injection volume and electrode distance on gene expression efficiency. (A) Relationship between the TP voltage level and the survival rate (dashed line) or gene transfer rate (solid line), 1 day after electroporation. After $200 \mathrm{nl}$ of plasmid solution was injected into the brain, five TPs of 10,30 , or $50 \mathrm{~V}$, of $50 \mathrm{~ms}$ duration, were applied at $950 \mathrm{~ms}$ intervals, after the single PP of $100 \mathrm{~V}$ of $0.1 \mathrm{~ms}$ duration. (B) Relationship between the TP voltage and the numbers of eGFP positive cells in individual crickets. Electroporation electrodes 
were placed at the base of each antenna. Data were acquired from the same set of samples used in (A), but the samples without eGFP fluorescence were discarded from the data set. (C) Numbers of eGFP positive cells when 20 or $200 \mathrm{nl}$ of plasmid solution were injected. Pulse voltages for the electroporation were $100 \mathrm{~V}$ for PP and $50 \mathrm{~V}$ for TPs. (D) Number of eGFP positive cells when the distance between the electrodes was approximately $1 \mathrm{~mm}$ or $2 \mathrm{~mm}$. The volume of plasmid solution was $200 \mathrm{nl}$ and the PP or TP voltages were $100 \mathrm{~V}$ and $50 \mathrm{~V}$, respectively.

Fig. 3. Distribution of eGFP positive cells. The injection site and the electrode layout were aligned to target the left mushroom body (A), antennal lobe (B, C) or the left hemisphere of the protocerebrum (D). Localization of eGFP expression was attempted by reduction of the plasmid volume (20 nl, in A-C) and/or by unilateral arrangement of the electrodes (C and D). Upper panels are superimposed drawings of the three samples in different colors, each of which shows the location of eGFP positive cells in relation to the brain outline. The targeted injection site is indicated by $\mathrm{X}$ and the placement of the positive and negative electrodes is indicated by circled + and - marks, respectively. The fluorescent images in lower panels are typical samples from each protocol. Fluorescent images in A2, B2 and C2 are frontal views, whilst D2 is a rear view of the brain. Scale bars are $250 \mu \mathrm{m}$.

Fig. 4. Fluorescent images of samples damaged by electrodes in contact with the brain surface. Localized gene expression was targeted to the central region (A) or the left hemisphere (B, C, D) of the brain. Plasmid solution (200 nl) was injected into the target sites, indicated by X. Voltages of PP and TPs are shown in the upper right of each image and the electrode placement is indicated by circled + and - . As shown by dashed lines in $\mathrm{A}-\mathrm{C}$, the damaged tissue, indicated 
by discoloration, was observed in the central part of the brain. All fluorescent images show the frontal view of the brain. Scale bars are $250 \mu \mathrm{m}$.

\section{Fig. 5.}

Expression of Yellow Cameleon 3.60 and Channel Rhodopsin $2(\mathrm{ChR} 2)$ within the cricket brain. (A, B) Fluorescent images of a cricket brain 4 days after the introduction of YC3.60 (A) and 8 days after the introduction of ChR2-DsRed2 (B), using electroporation. Arrow heads indicate somata of ChR2 positive cells. (C) Relationship between number of YC3.60 positive cells and time after electroporation.

\section{Table 1}

Number of animals that displayed various viability statuses after electroporation with different TP voltages. The number within the brackets of the $50 \mathrm{~V}$ cells indicates injection volume (200 or $20 \mathrm{nl}$ ). Electroporation was applied with the electrodes placed at the base of each antenna. 
A1

B1

(4)

$20 \mathrm{nl}$

$100 \mathrm{~V} / 50 \mathrm{~V}$

$\underline{\underline{=}}$

$\mathrm{A} 2$

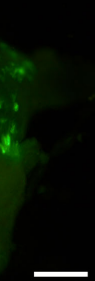

$20 \mathrm{nl}$

$100 \mathrm{~V} / 50 \mathrm{~V}$

$=$

B2

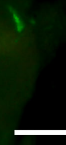

$20 \mathrm{nl}$

$100 \mathrm{~V} / 50 \mathrm{~V}$

C2

芶

$\underline{\underline{2}}$

$25 \mathrm{~V} / 12.5 \mathrm{~V}$

D2

$\checkmark$

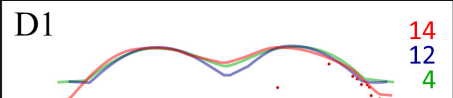

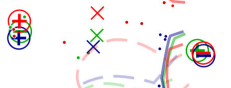

용

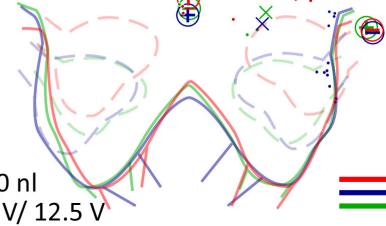




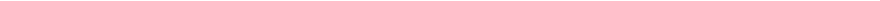


C

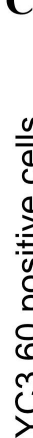

$\checkmark$

$\checkmark$

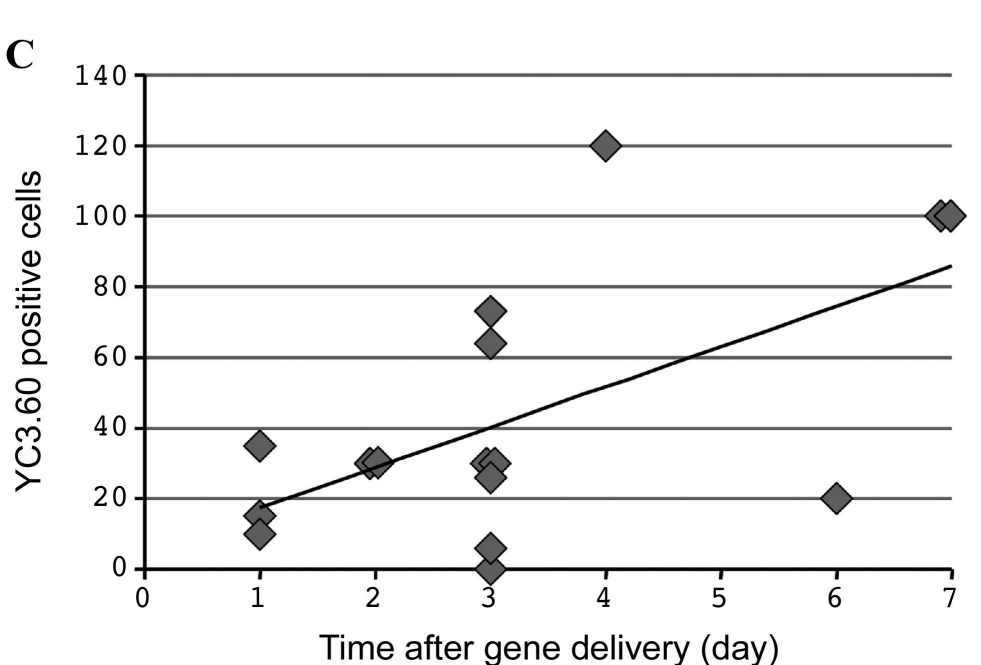

